\title{
Optical angular momentum conversion in a nanoslit
}

\author{
Philip F. Chimento, ${ }^{1, *}$ Paul F. A. Alkemade, ${ }^{2}$ Gert W. 't Hooft, ${ }^{1,3}$ and Eric R. Eliel ${ }^{1}$ \\ ${ }^{1}$ Leiden University, Huygens Laboratory, P.O. Box 9504, 2300 RA Leiden, Netherlands \\ ${ }^{2}$ Delft University of Technology, Kavli Institute of Nanoscience, Lorentzweg 1, 2628 CJ Delft, Netherlands \\ ${ }^{3}$ Philips Research Laboratories, Prof. Holstlaan 4, 5656 AA Eindhoven, Netherlands \\ ${ }^{*}$ Corresponding author: chimento@physics.leidenuniv.nl
}

Received October 9, 2012; accepted October 18, 2012;

posted October 24, 2012 (Doc. ID 176976); published November 28, 2012

\begin{abstract}
We demonstrate partial conversion of circularly polarized light into orbital angular momentum-carrying vortex light with opposite-handed circular polarization. This conversion is accomplished in a novel manner using the birefringent properties of a circular subwavelength slit in a thin metal film. Our technique can be applied over a very wide range of frequencies and even allows the creation of anisotropic vortices when using a slit without circular symmetry. (c) 2012 Optical Society of America
\end{abstract}

OCIS codes: $310.6628,230.7370,260.1440,050.1930,240.6680,050.4865$.

The curious phenomenon of optical vortices arising from axial symmetry in birefringent materials has been studied in uniaxial crystals of variable length $[\underline{1}, 2]$ and birefringent plates with a spatially varying optical axis and half-wave retardation (" $q$-plates") [3-5]. This interaction between spin and orbital angular momentum of light by way of a Berry-Pancharatnam phase has also been studied in space-variant gratings [] $]$ and plasmonic nanostructures in the context of selection rules [7], and also outside the domain of optics, in electron beams [8]. We present here a novel method for this conversion using a subwavelength slit in a metal film that acts like a quarter-wave plate [9]. We show how this method relaxes the requirement of circular symmetry, allowing greater versatility in the form of the vortex created.

In optics, a spin angular momentum of $\pm \hbar$ is associated with a circularly polarized photon. Orbital angular momentum is often associated with an optical vortex beam, where the phase increases azimuthally around the optical axis. These beams have a topological charge $Q$, equal to the number of full cycles the phase makes in one trip around the optical axis. The expectation value of the orbital angular momentum per photon is $Q \hbar$ [10]. The difference between the two forms of angular momentum is beautifully apparent in the interaction of a beam with small particles: interaction with the spin angular momentum in the absence of absorption requires particles that are birefringent; they will start to rotate about their own axis, whereas interaction with a beam carrying orbital angular momentum causes particles, whether birefringent or not, to rotate about the beam's optical axis [11].

Recently, we reported on how a subwavelength slit in a metal film can act as an optical retarder [9]. A slit that is subwavelength in one direction and extended in the other has two eigenpolarizations: parallel and perpendicular to the slit. By careful design of the slit's width and depth, it is possible to construct a slit that behaves like a quarter-wave retarder for incident light of a certain wavelength, with its fast axis (i.e., with the lowest index of refraction) parallel to the orientation of the slit. One can achieve similar results using subwavelength structures with different resonances for orthogonal polarization components $[\underline{12}, \underline{13}]$.

Illuminating the straight slit with circular polarization results in linear polarization being transmitted. The associated change in angular momentum means that a torque is exerted on the sample [14]. For a circular slit, the waveplate axes' orientations vary along the slit so that it acts as a space-variant quarter-wave plate. In this circularly symmetric configuration, photonic spin angular momentum cannot be transferred to the sample, and must be converted to photonic orbital angular momentum in order to conserve the total angular momentum. This intuitive picture is confirmed by calculating the expectation value per photon of the spin $(S)$ and orbital $(L)$ angular momenta, averaged over the whole beam [15]. Whereas the input state has $S=\hbar, L=0$, the output state has $S=0, L=\hbar$. The total angular momentum is indeed conserved.

To confirm this by experiment, we took a glass substrate of $0.5 \mathrm{~mm}$ thickness. On it we deposited a titanium adhesion layer of $10 \mathrm{~nm}$ thickness, and on that a gold film of $200 \mathrm{~nm}$ thickness. We milled a circular slit, $20 \mu \mathrm{m}$ in diameter and $(180 \pm 10) \mathrm{nm}$ wide, through the gold film using a focused $\mathrm{Ga}^{+}$ion beam. Figure $\underline{1(\mathrm{c})}$ shows a

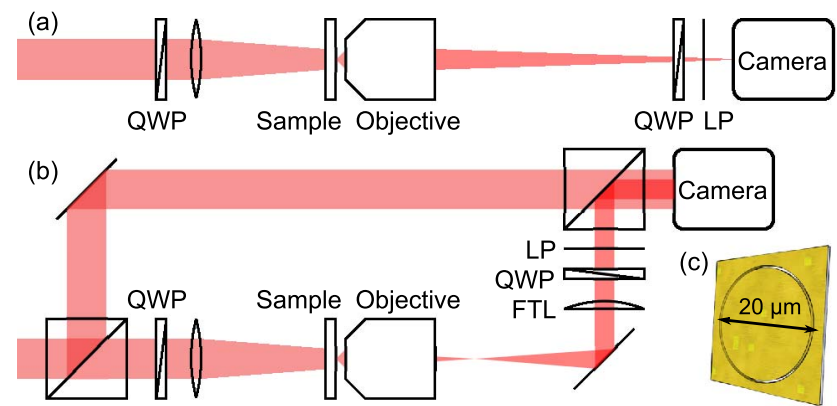

Fig. 1. (Color online) Sketches of the experimental setups. QWP, quarter-wave plate; FTL, Fourier-transforming ( $2 f$ ) lens; and LP, linear polarizer. (a) Setup used to image the ring slit. The quarter-wave plate and linear polarizer on the right-hand side of the figure measure the local polarization state of the light. (b) Setup used to measure the polarization and phase of the far field of the slit. The objective's focus is now not on the camera but in the focus of the FTL. In this case, the quarter-wave plate and polarizer are simply used to view the $\hat{\boldsymbol{\sigma}}_{+}$and $\hat{\boldsymbol{\sigma}}_{-}$components separately. An interferometer measures the phase of each polarization component. When not measuring the phase, we simply block the reference beam. (c) Sketch of the structure milled into the sample. 
sketch of the structure. We conducted the experiment using a diode laser $(\lambda=830 \mathrm{~nm})$. We used a quarterwave plate to give the laser beam a circular polarization state, $\hat{\boldsymbol{\sigma}}_{+}$. (We define $\hat{\boldsymbol{\sigma}}_{ \pm}=(\hat{\mathbf{x}} \pm i \hat{\mathbf{y}}) / \sqrt{2}$.) We then focused the beam weakly onto the glass side of the sample. The beam diameter at the waist was $90 \mu \mathrm{m}$, so that, effectively, the $20 \mu \mathrm{m}$ structure was illuminated with a plane wave. We used a microscope objective (NA 0.4) to image the slit onto a CCD camera (Apogee Alta U1).

We measured the transmitted polarization as a function of the transverse position within the image. To determine this polarization, we used a linear polarizer and a quarter-wave plate, as shown in Fig. 1(a), from which we extracted the normalized Stokes parameters $s_{i}=S_{i} / S_{0}$ according to the method described in [16] as a function of position. Figure 2 shows the results of this experiment. We observe small variations in the transmitted intensity along the ring, which are probably caused by small variations in the slit width related to the grain structure of the metal film. The transmitted polarization, however, shows excellent agreement with our calculations.

The polarization measured in Fig. 2 suggests that the transmitted light has azimuthally dependent polarization. Such beams, usually called vector beams, were first described as waveguide modes [17] with a dark spot in the center due to a polarization singularity. At first glance, one might expect our nanostructure to produce a vector beam, and thus have a dark spot in the center of the far field. However, calculating the far field by numerical Fourier transform shows that there is no dark spot in the center; in fact, the local polarization on the optical
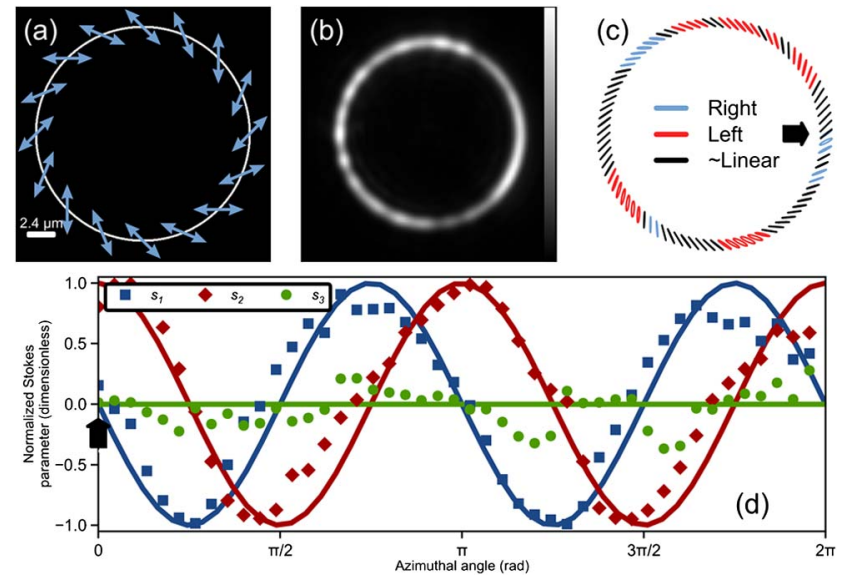

Fig. 2. (Color online) (a) Diagram showing the expected local polarization state of light transmitted through the ring slit. The transmitted intensity is constant over the slit. (b) Measured transmitted intensity. (c) Local polarization ellipses of the transmitted light. Light grey ellipses indicate right-handed elliptical polarization, dark grey ones indicate left-handed elliptical polarization, and black lines indicate polarization states with ellipticity less than 10\%. (d) Measured normalized Stokes parameters of the light transmitted through the ring slit as a function of azimuthal angle. This shows the same information as (c), but here it is easier to compare it to the expected results (solid curves), with which we observe quite good agreement. An angle of $0^{\circ}$ corresponds to 3 o'clock in (c) (see arrows), and increases counterclockwise. axis in the far field is purely $\hat{\boldsymbol{\sigma}}_{+}$, the same as the input polarization.

In order to explore this further, we derived an analytical expression for the far field by Fourier-transforming the field shown in Fig. 2(a) and linearizing over the slit width $\Delta R$,

$$
\mathbf{E}_{0}^{\mathrm{FF}} \approx \frac{1+i}{\sqrt{2}} \pi R_{0} \Delta R\left[J_{0}\left(R_{0} k_{\perp}\right) \hat{\boldsymbol{\sigma}}_{+}-i \exp (2 i \theta) J_{2}\left(R_{0} k_{\perp}\right) \hat{\boldsymbol{\sigma}}_{-}\right],
$$

where $R_{0}$ is the radius of the ring, $k_{\perp}$ the transverse component of the wave vector, and $J_{n}$ denotes the Bessel function of the first kind of order $n$. This expression is valid for small $\Delta R$ in the paraxial approximation. These fields are visualized in Figs. 3(a) and 3(d). Note that the characteristic length scale in the far field is given by the radius $R_{0}$ of the circular structure; that is, the diffraction pattern does not arise from an aperture cutoff, but from the interference between opposite points on the circular slit.

This expression indicates that half of the transmitted beam energy has been converted from the $\hat{\boldsymbol{\sigma}}_{+}$to the $\hat{\boldsymbol{\sigma}}_{-}$state, while acquiring a topological charge of +2 . (The integral of any $J_{n}(x)$ to infinite $x$ is equal to 1 if $n \geq 0$.) This acquisition of topological charge by the opposite-handed component of the transmitted beam can be seen as the result of spin-to-orbital angular momentum conversion, but it is equally instructive to consider it a Berry-Pancharatnam phase, the result of traveling from the north pole $\left(\hat{\boldsymbol{\sigma}}_{+}\right)$of the Poincaré sphere to the south pole $\left(\hat{\boldsymbol{\sigma}}_{-}\right)$through all possible points on the equator, twice.

We performed further experiments to explore this, using a $2 f$ system to examine the far field; see
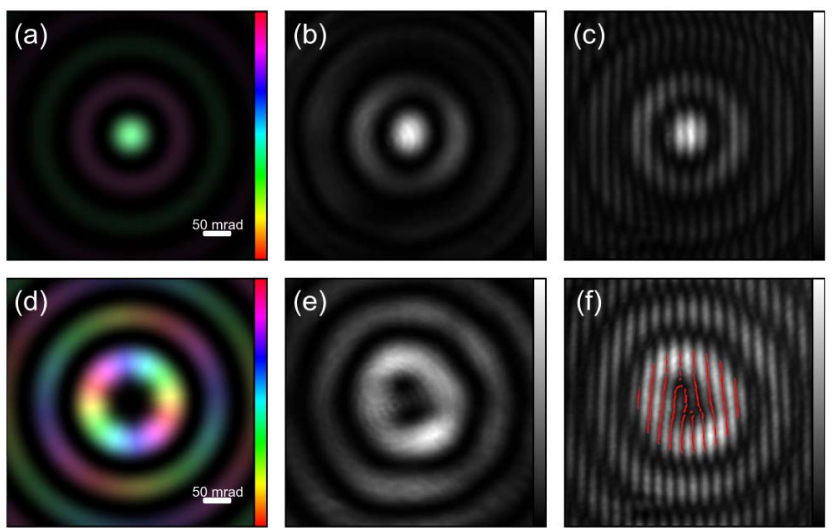

Fig. 3. (Color online) Far-field diffraction pattern of the circular slit, split into $\hat{\sigma}_{+}$(top) and $\hat{\sigma}_{-}$(bottom) polarized components. (a), (d) Calculated intensity and phase in the far field; the luminance indicates the intensity, and the hue indicates the phase, cycling from 0 to $2 \pi$. The $\hat{\sigma}_{-}$component has a topological charge of 2. (b), (e) Measured intensity of both components, showing good agreement with the calculations. (c), (f) Interferograms using reference beams with the appropriate circular polarization, demonstrating the phase of both components. In (f), one fringe splits into three, indicating a helical wavefront with a topological charge of 2 , as in the calculations. The interference fringe minima are marked with lines for clarity, using the technique described in [19]. 
Fig. 1(b). We used a quarter-wave plate and a linear polarizer to measure the intensity distribution of the $\hat{\boldsymbol{\sigma}}_{+}$and $\hat{\boldsymbol{\sigma}}_{-}$components of the far field separately. We also used a misaligned Mach-Zehnder interferometer to visualize the phase of the light transmitted through the slit. The interference pattern consists of parallel interference fringes, which fork according to the topological charge carried by the beam [18]. Figure $\underline{3}$ shows the results of our measurements compared to the calculation of Eq. (1). The interferograms in Figs. 3(c) and 3(f) show that the $\hat{\boldsymbol{\sigma}}_{-}$component does indeed have a topological charge of +2 , whereas the $\hat{\boldsymbol{\sigma}}_{+}$component carries no topological charge.

We also consider what happens when the amplitudes of the transmitted polarization components are unequal, or when the retardation is not exactly a quarter wave. One can easily derive that the polarization conversion efficiency $\eta$ is independent of the slit's dichroism but depends on the relative phase retardation $\Delta \phi$ between the polarization components as follows:

$$
\eta=I_{-} / I_{\text {total }}=\sin ^{2}(\Delta \phi / 2),
$$

where $I_{-}$is the intensity of the $\hat{\sigma}_{-}$component. If the slit were to behave like a half-wave retarder, then $\eta$ would become unity. However, designing a half-wave-like slit would once again require careful research to find a suitable width, depth, and material.

This last result suggests that optical spin-orbit conversion is a universal property of a circular nanoslit as long as the local polarization eigenmodes have different propagation constants but similar damping. In order to obtain full conversion efficiency, one obviously has to adjust the properties of the slit to the wavelength of the incident light in a way similar to the design of a liquid-crystal-based $q$-plate [3] for a certain wavelength. An attractive benefit of our slit approach to optical spinorbit conversion is that it is universal; i.e., it can be used at wavelengths from the deep UV to the far infrared.

One may wonder what happens when the metallic nanoslit is no longer cylindrically symmetric but still encircles a singly connected domain. Since the circular symmetry is broken, angular momentum transfer to the sample is no longer forbidden. Then, for a quarterwave-like slit with a circularly polarized Gaussian beam incident on it, half of the transmitted light will have opposite circular polarization and carry a charge two vortex with a broad orbital angular momentum spectrum. Contrary to the case of a circular slit, this vortex will be anisotropic.

In conclusion, we have demonstrated spin-to-orbital angular momentum conversion of an electromagnetic field upon transmission through a circular metallic nanoslit. When illuminated with circularly polarized light, part of the field transmitted through the slit is converted to the opposite handedness and its topological charge is increased or decreased by 2 , corresponding to a conversion of spin angular momentum to orbital angular momentum. The conversion efficiency is a function of the relative phase delay that the slit imposes on orthogonal polarization components. This means that full spinorbit conversion could be achieved simply by passing the light through a half-wave-like slit in a thin metal film. Using a closed-loop slit without circular symmetry, on the other hand, opens up a new world of possibilities for creating anisotropic optical vortices.

This work is part of the research program of the Foundation for Fundamental Research on Matter (FOM), which is part of the Netherlands Organisation for Scientific Research (NWO). This work was also supported by PHORBITECH, which is part of the Future and Emerging Technologies (FET) program within the 7th Framework Program for Research of the European Commission.

\section{References}

1. A. Ciattoni, G. Cincotti, and C. Palma, J. Opt. Soc. Am. A 20, 163 (2003).

2. E. Brasselet, Ya. Izdebskaya, V. Shvedov, A. S. Desyatnikov, W. Krolikowski, and Yu. S. Kivshar, Opt. Lett. 34, 1021 (2009).

3. L. Marrucci, C. Manzo, and D. Paparo, Phys. Rev. Lett. 96, 163905 (2006).

4. E. Karimi, B. Piccirillo, L. Marrucci, and E. Santamato, Opt. Lett. 34, 1225 (2009).

5. E. Brasselet and C. Loussert, Opt. Lett. 36, 719 (2011).

6. Z. Bomzon, V. Kleiner, and E. Hasman, Opt. Lett. 26, 1424 (2001).

7. Y. Gorodetski, N. Shitrit, I. Bretner, V. Kleiner, and E. Hasman, Nano Lett. 9, 3016 (2009).

8. E. Karimi, L. Marrucci, V. Grillo, and E. Santamato, Phys. Rev. Lett. 108, 044801 (2012).

9. P. F. Chimento, N. V. Kuzmin, J. Bosman, P. F. A. Alkemade, G. W. 't Hooft, and E. R. Eliel, Opt. Express 19, 24219 (2011).

10. L. Allen, M. W. Beijersbergen, R. J. C. Spreeuw, and J. P. Woerdman, Phys. Rev. A 45, 8185 (1992).

11. A. T. O'Neil, I. MacVicar, L. Allen, and M. J. Padgett, Phys. Rev. Lett. 88, 053601 (2002).

12. A. Roberts and L. Lin, Opt. Lett. 37, 1820 (2012).

13. P. Genevet, N. Yu, F. Aieta, J. Lin, M. A. Kats, R. Blanchard, M. O. Scully, Z. Gaburro, and F. Capasso, Appl. Phys. Lett. 100, 013101 (2012).

14. R. A. Beth, Phys. Rev. 50, 115 (1936).

15. M. V. Berry, M. R. Jeffrey, and M. Mansuripur, J. Opt. A 7, 685 (2005).

16. B. Schaefer, E. Collett, R. Smyth, D. Barrett, and B. Fraher, Am. J. Phys. 75, 163 (2007).

17. E. A. J. Marcatili and R. A. Schmeltzer, Bell Syst. Tech. J. 43, 1783 (1964).

18. I. V. Basistiy, M. S. Soskin, and M. V. Vasnetsov, Opt. Commun. 119, 604 (1995).

19. L. Cai, Q. Liu, and X. Yang, Opt. Laser Technol. 35, 295 (2003). 